\title{
Des images en débat : de la blessure de Geneviève Legay à la répression des Gilets Jaunes
}

Images in Debate: From the Injuring of Geneviève Legay to Police Repression of

"Yellow Vests"

\section{Édouard Bouté et Clément Mabi}

\section{OpenEdition} Journals

Édition électronique

URL : https://journals.openedition.org/edc/9996

DOI : 10.4000/edc.9996

ISSN : 2101-0366

Éditeur

Université de Lille

\section{Édition imprimée}

Date de publication : 1 juillet 2020

Pagination : 29-52

ISBN : 978-2-917562-23-9

ISSN : $1270-6841$

\section{Référence électronique}

Édouard Bouté et Clément Mabi, « Des images en débat : de la blessure de Geneviève Legay à la répression des Gilets Jaunes », Études de communication [En ligne], 54 | 2020, mis en ligne le 02 janvier 2022, consulté le 08 janvier 2022. URL : http://journals.openedition.org/edc/9996 ; DOI : https:// doi.org/10.4000/edc.9996 


\section{Des images en débat : de la blessure de Geneviève Legay à la répression des Gilets Jaunes}

Images in Debate: From the Injuring of Geneviève Legay to Police Repression of "Yellow Vests"

Édouard Bouté

UTC - Université de Technologie de Compiègne, Laboratoire Costech - UPR 2223 - Connaissance Organisation et Systèmes TECHniques edouard.boute@utc.fr

Clément Mabi

UTC - Université de Technologie de Compiègne, Laboratoire Costech - UPR 2223 - Connaissance Organisation et Systèmes TECHniques clement.mabi@utc.fr 
Résumé / Abstract

Cet article étudie la mobilisation des images dans les débats Twitter concernant " l'affaire Geneviève Legay ", militante blessée lors d'une manifestation Gilets Jaunes interdite à Nice le 23 mars 2019. Notre enquête montre une évolution du statut des images dans les échanges. D'abord utilisées comme appuis à l'argumentation pour justifier le point de vue exprimé, elles deviennent progressivement le support d'un affrontement de principe, moral et axiologique, dont l'objectif est moins d'éclairer un point de vue sur l'événement en lui-même que de le réinscrire dans des enjeux et un conflit plus larges concernant la légitimité de l'action policière en situation de maintien de l'ordre. Nous faisons I'hypothèse que ce glissement s'explique en partie par la configuration sociotechnique et technosémiotique des RSN qui structures la dynamique conflictuelle de l'affaire. Les formats d'écritures orientés vers l'interaction entre internautes et le partage d'images contribueraient à la montée en généralité du conflit et à l'ouverture du cadrage des échanges en fournissant de nouveaux appuis à l'argumentation.

Mots-clés : répression policière, gilets jaunes, images, débats, Twitter.
This article examines the mobilization of images in Twitter debates surrounding the activist Geneviève Legay, injured during a banned "Yellow Vest" demonstration in Nice on March $23^{r d}, 2019$. Our investigation shows a gradual change in the status of images. Initially used to bolster differing points of view, images become a medium for confrontation of moral and axiological principles, with the objective of integrating this event into broader issues and conflicts concerning the legitimacy of police action in a policing situation. We hypothesize that the reason for this shift resides partly in the sociotechnical and techno-semiotic configuration of social networking sites, which structures the conflictual dynamics of the case. Writing formats oriented towards interaction between Internet users and the sharing of images contribute to the broadening of the conflict and the reframing of exchanges.

Keywords: police repression, public sphere, images, debates, Twitter. 
Le 23 mars 2019, Geneviève Legay, retraitée âgée de 73 ans, militante auprès de l'organisation Attac, est grièvement blessée à la tête lors d'une manifestation « Gilets Jaunes » interdite à Nice. Rapidement, plusieurs interprétations de l'événement se font face. Pour certain·es, cette blessure serait une nouvelle bavure policière, dans un contexte où la stratégie de maintien de l'ordre face au mouvement social en cours est vivement contestée'. Pour d'autres, Geneviève Legay se serait blessée " toute seule " et n'aurait tout simplement pas dû se trouver sur place. Dans les jours et les semaines qui suivent, le débat est régulièrement alimenté par les prises de position d'acteurs publics. Dès le 25 mars, Nice-Matin rapporte les propos du chef de l'État, Emmanuel Macron, qui déclare que Geneviève Legay «n'a pas été en contact avec les forces de l'ordre $^{2}$ ». Le même jour, le procureur de la République de Nice écarte lui aussi toute possibilité de "contact avec un agent de sécurité ${ }^{3}$ » et tente ainsi de clôturer l'affaire. Pourtant, le 29 mars, ce dernier revient sur sa déclaration initiale en affirmant que " contrairement à ce qui pouvait être interprété des premières images, il y a eu un contact direct entre un membre des forces de l'ordre et Mme Legay ". Le 31 mars, le policier concerné, qui s'est reconnu sur les images de vidéosurveillance au moment de la charge policière, admet avoir poussé la militante ${ }^{5}$.

Rapidement, I'affaire autour de la responsabilité policière dans la chute de la militante est devenue " triadique » (Lemieux, 2007), chaque camp tentant d'enrôler l'opinion publique afin de convaincre du bien-fondé de sa lecture de l'événement. Dans cette bataille interprétative, les images et les vidéos ont joué un rôle décisif. Sur les réseaux sociaux numériques (RSN), comme Facebook ou Twitter, les internautes ont mobilisé des contenus issus des médias traditionnels pour appuyer leur propos, reconstruire le déroulé des faits et attribuer d'éventuelles responsabilités.

Dans cet article, nous proposons d'analyser en détail les processus de circulation de ces contenus médiatiques afin de mieux saisir les processus de construction documentaire de l'argumentation dans l'affaire Legay. Notre enquête montre une évolution du statut des images. D'abord principalement utilisées comme appuis à l'argumentation pour justifier le point de vue exprimé,

1 Collectif, "TRIBUNE. 'Nous assistons à une volonté délibérée de nous empêcher de travailler' : plus de 300 journalistes dénoncent les violences policières", sur le site web de Franceinfo (1er mai 2019).

2 Denis Carreaux, "Chine, bouchons, militante blessée, hôtel de police à Nice... Emmanuel Macron se confie en exclusivité à Nice-Matin », sur le site web de Nice-Matin (25 mars 2019).

3 "'Aucun contact' entre la manifestante blessée à Nice et un policier ", sur le site web d'Europe1 (25 mars 2019).

4 "Nice : la manifestante a bien été poussée par un policier », sur le site web de Franceinfo (29 mars 2019).

5 "Le policier qui a poussé Geneviève Legay exprime ses 'sincères regrets' ", sur le site web du Parisien (31 mars 2019). 
elles deviennent progressivement le support d'un affrontement de principe, moral et axiologique, dont l'objectif est moins d'éclairer un point de vue sur l'événement en lui-même que de le réinscrire dans des enjeux et un conflit plus larges concernant la légitimité de l'action policière en situation de maintien de I'ordre. Nous faisons I'hypothèse que ce glissement s'explique en partie par la configuration sociotechnique et technosémiotique des RSN qui structure la dynamique conflictuelle de l'affaire. Les formats d'écritures orientés vers l'interaction entre internautes et le partage d'images contribueraient à la montée en généralité du conflit et à l'ouverture du cadrage des échanges en fournissant de nouveaux appuis à l'argumentation.

\section{1. \\ Les RSN comme levier d'une nouvelle culture du débat public}

Le plein essor d'Internet et du web au tournant des années 2000 a facilité la démocratisation de l'espace public ${ }^{6}$ en permettant à chacun.e de publier ses propres contenus et de les diffuser. Les nouvelles pratiques communicationnelles liées à ces innovations technologiques témoignent de l'avènement d'une " culture du numérique » caractérisée par des valeurs comme la participation, la collaboration, la transparence et des pratiques parfois qualifiées de «bricolage » (Jenkins, 2006 ; Cardon, 2019). Dominique Cardon estime ainsi que le centre de gravité des démocraties occidentales se situerait désormais dans une "démocratie Internet » (2010), où Internet constitue un espace d'expérimentation démocratique pour des individus émancipés des nombreuses contraintes matérielles inhérentes à la prise de parole en public et capable de s'exprimer sans attendre l'autorisation des autorités et des gatekeepers traditionnels (politiques, journalistes...).

De nombreux travaux se sont intéressés aux effets politiques de ce basculement, en s'intéressant notamment à l'appropriation par les mouvements sociaux des technologies numériques, y voyant un renouvellement des formes de " médiactivisme " qui mettent à profit les formes d'expression variées (témoignage subjectif, conversation...) ainsi rendues possibles (Cardon et Granjon, 2010). Leur capacité à favoriser la production de discours contrehégémoniques, susceptibles de renouveler la critique des médias traditionnels et de renverser la « hiérarchie du voir » imposée par les espaces publics dominants a également été pointée (Voirol, 2005 ; Granjon, 2018). Ces discours contre-hégémoniques se structurent au sein de communautés interprétatives

6 L'espace public est ici considéré dans la perspective d'Habermas et de ses critiques. Nous le considérons comme espace de circulation de l'information, de constitution d'une opinion politique et d'expression publique de cette opinion (Habermas, 1962 ; Fraser, 2001 [1992] notamment). 
différenciées où les échanges s'agrègent jusqu'à faire advenir des visions du monde partagées par les participant·es qui s'y forgent une grille de lecture de l'actualité (Carbou 2015b ; Campion, 2016). Ainsi, loin de l'idéal d'un espace public unifié, la "démocratie Internet " se déploie dans une multitude d'espaces que l'on peut qualifier "d'arènes » de débat ${ }^{7}$. Ces arènes prennent forme dans une " multitude d'espaces symboliques et matériels où se réalisent des discussions entre différents acteurs et groupes sociaux, tels que [...] les forums sur Internet » (Dalibert et al., 2016, 8).

L'organisation de ces échanges fait l'objet de nombreuses controverses. On leur reproche leur forte conflictualité (Badouard, 2017) ou encore leur capacité à renforcer l'entre-soi des discussions (Sustein, 2009 ; Pariser, 2011). Cet effet « chambre d'écho » rend compte du fait qu'au sein de ces arènes, la majorité des participant.es partage les mêmes analyses et dispose des mêmes informations, ce qui a pour effet de créer un " dialogue de sourds » (Angenot, 2008) où chacun cherche à faire valoir son point de vue, sans véritablement prendre en considération les arguments contradictoires.

Pour mieux comprendre cette nouvelle culture du débat public, des travaux ont exploré une hypothèse " sociotechnique " ou " technosémiotique " qui interroge le rôle de la matérialité des processus de communication. II s'agit alors de considérer l'importance du design des dispositifs, de leur configuration technique, dans la structuration des échanges. Le rôle structurant des algorithmes dans l'organisation de la visibilité des points de vue exprimés est régulièrement mis en avant pour expliquer que certains contenus, notamment les plus sollicités, gagneraient artificiellement en audience et donc en popularité (Gillespie, 2014 ; Jammet, 2018). Dans son ouvrage, The revolution that wasn't. How digital activism favors conservatives, Jen Schradie (2019) démontre que les messages les plus simples et clivants sont ceux qui s'adaptent le mieux aux contraintes de la visibilité algorithmique. Son enquête montre que la " démocratie Internet " a tendance à renforcer le poids des mobilisations conservatrices et la diffusion de leurs messages radicaux. Stefania Milan (2013), à travers le concept de "cloud protesting ", rappelle combien les usages des technologies numériques, leurs imaginaires et leurs représentations (réunis au sein du concept de cloud) ont contribué à façonner les discours produits par des mouvements sociaux récents, leurs organisations et la manière dont les participant·es se perçoivent au sein des mouvements.

Dans le prolongement des travaux de Juliette Rennes (2007) qui considère les "prédilections argumentatives » des différentes arènes pour insister sur le fait qu'en fonction du contexte d'énonciation, un argument ne sera pas

7 La notion d'arène est mobilisée ici dans une perspective pragmatiste pour qualifier les « dispositifs visant à mettre en relation des locuteurs et des audiences auxquelles ils s'adressent " (Dodier, 1999, 109) où des sujets d'actualité sont débattus. 
formulé de la même manière, plusieurs recherches se sont intéressées à la façon dont les arènes numériques orientent la manière dont les arguments sont rendus visibles. Ainsi, formuler un message sur Twitter, en raison de ses contraintes en nombre de signes, de l'usage des mots-clés (hashtags), etc., ne permet pas aux locuteur.trices de se saisir d'un sujet de la même manière que sur YouTube ou Instagram, centrés sur la diffusion d'images. En fonction des formats de communication, différentes manières de faire sens avec le monde sont rendues possibles. Les travaux de Clément Mabi (2016) sur l'usage différencié et stratégique des espaces d'expression par les militant-es en lutte contre le projet d'aéroport à Notre-Dame-des-Landes et ceux de Virginie Julliard (2016) sur l'approche sémiotique des controverses autour de la " théorie du genre " sur Twitter permettent d'interroger les effets des dispositifs d'écriture sur les dynamiques des débats. Ainsi, le travail de Virginie Julliard ouvre des perspectives pour comprendre la manière dont Twitter structure l'écriture des publics, instrumente la circulation des textes et conditionne les processus d'interprétation. Elle montre notamment comment, grâce aux propriétés de Twitter (limite du nombre de caractères, possibilité de partager des images, des URL...), des communautés affectives se forment autour d'objets grâce à un travail d'éditorialisation des contenus. Ce travail sémiotique contribue à la mise en circulation d'émotions qui renforcent les clivages politiques.

L'enquête dont nous rendons compte dans cet article prolonge cette dynamique et interroge les processus de construction documentaire de l'argumentation en examinant le rôle des contenus sémiotiques mis en circulation sur les RSN et leur rôle dans l'affaire Legay. En effet, le web et la possibilité d'intégrer des contenus médiatiques à son expression en ligne favorisent la mobilisation de nombreux " appuis documentaires " à l'argumentation pour donner plus de force à son propos (Rebillard, 2017). Au sein de ces documents mobilisés, les images occupent une place centrale. André Gunthert les qualifie "d'images conversationnelles » pour expliquer que ces images partagées sont « susceptible[s] de fournir des matériaux robustes à la confrontation des opinions ». Ainsi, la vidéo, par exemple :

« [...] réunit plusieurs propriétés précieuses : celle d'être perçu[e] comme une convocation du réel, une présomption de témoignage, mais aussi une démonstration autosuffisante. [Elle] n'apporte pas seulement une information, [elle] constitue en [elle]-même un embrayeur d'interactions et un générateur de visibilité $[. .$.$] » (Gunthert, 2018, 133).$

Cette perspective est discutée dans la littérature. Des débats récents soulignent le fait qu'attribuer directement à l'image des propriétés susceptibles de susciter l'interaction risque de conduire à sous-estimer les asymétries de ressources des acteurs qui structurent les processus de visibilisation des images (Riboni, 2019). Le risque de sous-estimer le poids des structures industrielles et sociales qui contraignent la circulation de ces contenus a également été pointé (Douyère, 2019). En retraçant la circulation des images de l'affaire Legay 
sur Twitter, nous proposons ainsi de contribuer à cette réflexion et d'observer précisément ce que les caractéristiques technosémiotiques de ce RSN « font » aux débats en faisant évoluer le statut des images, à la fois « arguments » dans les échanges et « supports » aux débats.

\section{2. \\ Un cadrage médiatique favorable au discours officiel, contesté sur les RSN}

La blessure de Geneviève Legay a été fortement discutée et a fait l'objet d'une " bataille de cadres ", où différentes interprétations de l'incident se sont confrontées dans l'espace public. Dans un premier temps, les médias traditionnels (presse, télévision et radio) ont rendu compte de l'événement en revenant sur les faits, tout en proposant une première grille de lecture nécessairement partielle (Gamson et Modigliani, 1989). Prendre en compte cette dimension socialement construite du récit médiatique invite à considérer les productions présentées comme le résultat de la sélection de " quelques aspects d'une réalité perçue [...] afin de promouvoir une définition particulière d'un problème, une interprétation causale, une évaluation morale, et/ou une recommandation de traitement de l'objet décrit ${ }^{8}$ »(Entman, 1993, 52). Comme l'a bien montré la sociologie de l'action collective, imposer une définition, construire un problème et le rendre public, fait l'objet de luttes entre des entrepreneur.euses de causes, des institutions politiques et des relais médiatiques (Snow et al., 1986). Dans ces luttes, différentes dimensions du problème et visions du monde sont mises en avant pour décrire le problème. Nicolas Dodier (1999) parle de tensions "épistémo-politiques " pour qualifier ces dissensions en fonction "d'où parlent » les acteurs. Dans le cas présent, nous avons pu observer que la lecture de l'événement était fortement corrélée à leur positionnement relatif au mouvement social en cours, celui des Gilets Jaunes, et plus particulièrement à la controverse autour de l'usage de la force dans la stratégie de maintien de l'ordre.

Pour analyser ce cadrage, nous avons constitué via Europresse un corpus de 133 articles issus de la presse nationale (11 titres) et de Nice-Matin, publiés entre le 23 et le 29 mars 2019 (cf. Figure 1). 


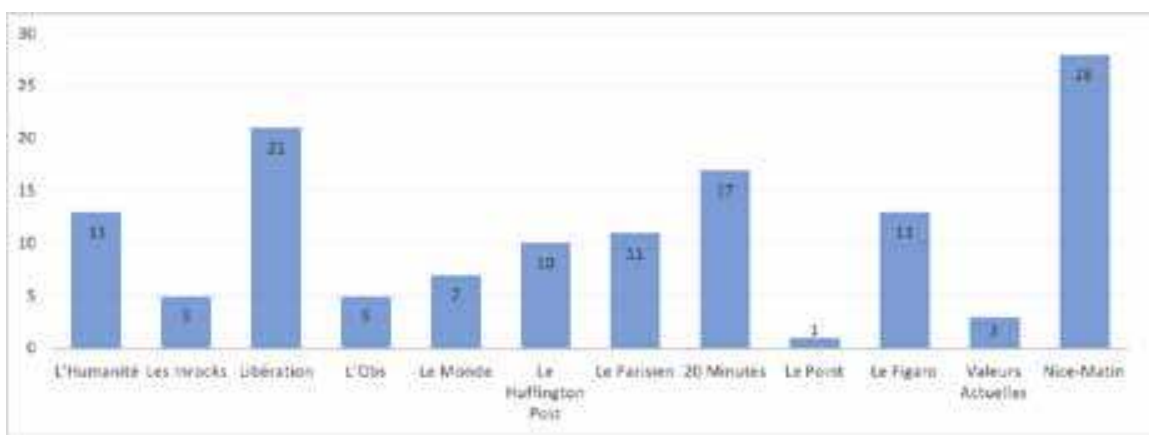

Figure 1 : Nombre d'articles publiés par les médias d'information de notre corpus

Ces articles relaient tous l'événement comme étant une blessure advenue pendant une charge policière. De manière générale, ils cherchent peu à prendre du recul sur les faits et se contentent de relayer les prises de position de différents acteurs. D'un côté, le discours officiel qui soutient l'absence de responsabilité ou d'illégitimité dans l'acte de la police (Emmanuel Macron, Christophe Castaner, le procureur de Nice, le préfet des Alpes-Maritimes, le maire de Nice, des député.es de la majorité, le policier incriminé...), de l'autre, un discours alternatif qui incrimine au contraire les forces de l'ordre (la famille de Mme Legay, leurs avocat·es, Attac, des "street medics ", des député·es Insoumis et communistes...). On observe cependant qu'au-delà d'un relai plutôt équitable des différentes positions, les cadrages diffèrent. Certains titres de presse, considérés à droite, comme Le Point ou Le Figaro, mais aussi 20 Minutes (31 articles du corpus), mettent en avant l'interdiction de manifester ou encore la violence de la manifestation des Gilets Jaunes le samedi précédent, afin de relativiser l'action des forces de l'ordre. Le Figaro écrit ainsi « que la manifestation du samedi 23 mars était 'illicite et interdite' et que l'action de dispersion des forces de l'ordre 'n'a pas pu surprendre qui que ce soit' ${ }^{9}$ ".

D'autres médias, plutôt classés à gauche comme L'Humanité, Libération ou Les Inrocks, mais aussi Le Huffington Post (49 articles), mettent au contraire en avant les paroles de celles et ceux qui revendiquent l'illégitimité de l'interdiction de manifester, ou qui mettent en doute la position officielle sur l'incident en insistant par exemple sur le calme du rassemblement, ou en se demandant si Geneviève Legay était vraiment dans le périmètre interdit lorsqu'elle a été percutée. Libération explique par exemple que Geneviève Legay « a lourdement 
chuté lors d'une charge de la police pour disperser la manifestation qui se déroulait dans le calme ${ }^{10} »$.

Notre enquête montre que rapidement les RSN se sont faits à leur tour l'écho de l'événement. Pour en analyser la diffusion, nous avons choisi d'étudier les dynamiques de circulation et d'interaction sur Twitter, ce RSN offrant une facilité d'accès au contenu pour les chercheur.es (Julliard, 2015) et ayant " acquis une place de choix comme moyen d'information sur l'actualité mais aussi comme support de mobilisation politique " (Rieder et Smyrnaios, 2012). Nous avons récolté, à l'aide du programme de scraping "Twint"1", 10329 tweets contenant la mention "Legay » dans le corps des messages entre le 23 mars et le 24 avril 2019. En analysant qualitativement ce corpus, nous avons pu réinscrire les tweets dans leur contexte d'énonciation initial, souvent des interactions, et ainsi suivre avec précision les pratiques des twittos.

Dans un premier temps, l'analyse de ce corpus de tweets nous a permis de relever que les comptes Twitter des médias d'information étudiés, massivement suivis pour la plupart (cf. Figure 2), ont largement partagé leurs articles concernant l'affaire Legay sur leurs propres comptes, contribuant ainsi fortement à la diffusion de ressources documentaires sur l'événement auprès d'un nombre important de twittos qui ont commenté, retweeté ou bien liké les tweets publiés (cf. Figure 3).

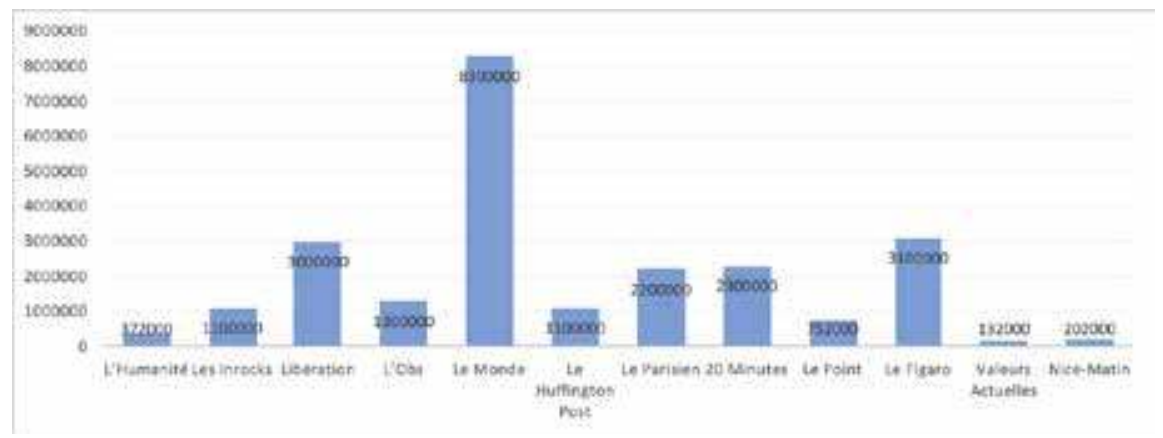

Figure 2 : Nombre de followers des médias d'information de notre corpus 


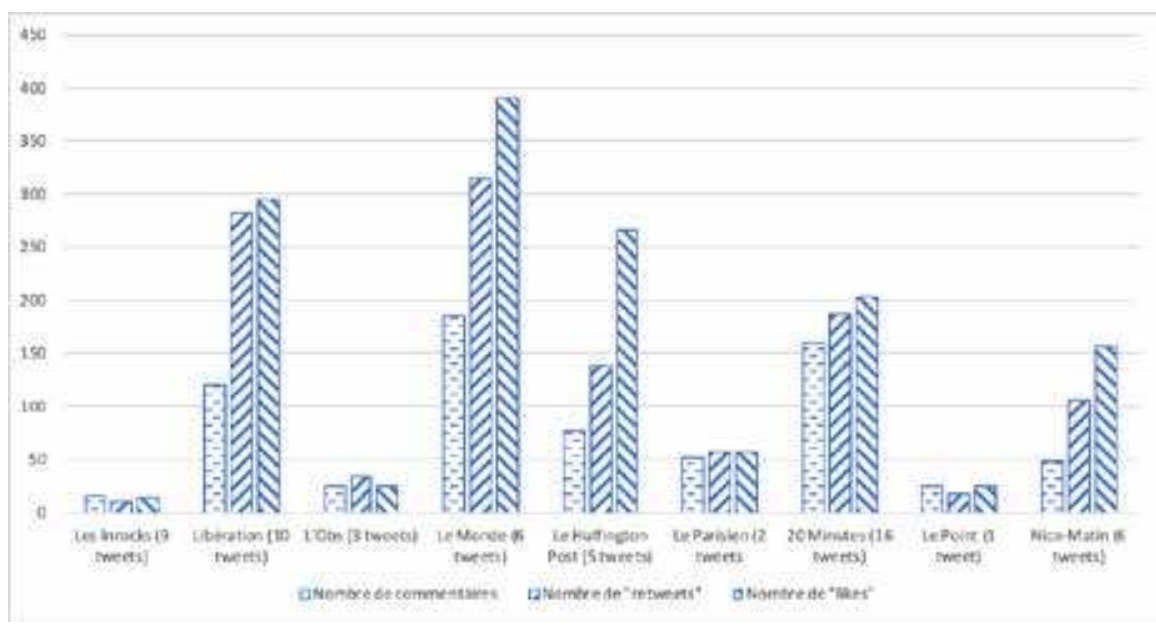

Figure 3 : Nombre de commentaires, retweets et likes des tweets des médias d'information de notre corpus entre le 23 et le 29 mars 2019

Dans un deuxième temps, notre observation montre que les internautes ont massivement mobilisé ces contenus médiatiques, profitant notamment des propriétés communicationnelles particulières de Twitter, favorables au partage de contenus médiatiques et d'images (Julliard, 2017). Sur les 10329 tweets étudiés, 7201 contiennent des URL, dont 3667 renvoient vers des articles de presse, des extraits télés ou radios. 978 tweets contiennent des photos et 176 des vidéos, pour la plupart initialement produites par les médias traditionnels. Effectivement, toutes les images de la chute de Mme Legay sont celles de professionnel-les de l'information, et non de militant·es ou de manifestant.es présent.es sur place. Si certain·es auteur·es mettent en avant l'importance des images amateures dans les luttes de visibilité sur Internet (Boëx, 2012 ; Riboni, 2015), nous constatons ici que les images produites par les médias traditionnels jouent un rôle structurant dans la mesure où elles bénéficient des importantes ressources en visibilité des institutions médiatiques.

Sur Twitter, comme dans la presse traditionnelle, différentes lectures de l'événement circulent. Mais dans cette arène, celles-ci sont plus radicales, conflictuelles et fragmentées, conformément à la nouvelle culture du débat public précédemment décrite. Dans un premier temps, les internautes ont cherché à mesurer la responsabilité de la police dans la chute de la militante en mobilisant des "preuves » censées justifier leur positionnement en reconstituant les circonstances de l'incident. Les twittos se sont alors appuyé·es sur les nombreuses images publiées par les médias, qui sont autant d'interprétations de la chute de la militante, qui ont circulé sur Twitter et qui ont été utilisées pour justifier leurs points de vue sur l'incident. Nous parlons "d'images-preuves » 
pour qualifier ces logiques d'appuis documentaires à l'argumentation des internautes.

Les premiers à se saisir d'images pour «faire preuve » sur Twitter sont les soutiens aux forces de l'ordre. Pour eux, la police n'a rien à voir dans la chute de la militante. Une image a été particulièrement mobilisée pour appuyer ce discours (cf. Figure 4). Geneviève Legay y occupe le premier plan, un drapeau arc-en-ciel barré du mot "PAIX » à la main. Elle est entourée d'autres manifestant·es en train de refluer face à l'avancée des policiers. Cette photo qui saisit l'instant précis de la chute ne permet effectivement pas, à elle seule, de déterminer si un policier est entré en contact avec elle, notamment en raison du drapeau qui masque une partie de la scène. La thèse qui réfute la bavure policière est par ailleurs étayée par la présence d'une autre manifestante sur l'image, vêtue d'un haut jaune fluo, qui consulte son téléphone portable sans sembler en danger.

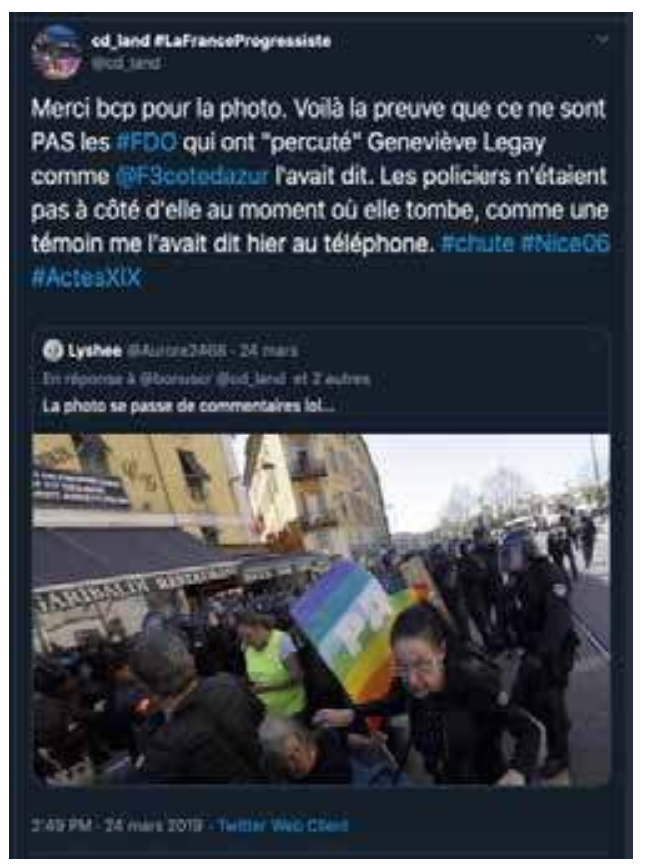

Figure 4 : L'image massivement utilisée dans les tweets des soutiens à la police

Le message associé à l'image par @cd_land laisse penser que cette image seule apporte la preuve de l'absence de contact entre Geneviève Legay et les forces de l'ordre (désignées sous le mot-clé \#FDO). L'internaute se place ainsi en contrepoint du discours officiel, notamment celui de France 3 Côté d'Azur. On retrouve ici la propension des technologies de communication numériques, 
et particulièrement les RSN, à faciliter la diffusion de lectures contre-hégémoniques des événements (Cardon et Granjon, 2010 ; Mabi, 2016).

C'est à partir du 25 mars, date des déclarations d'Emmanuel Macron puis du procureur de Nice en défense des forces de l'ordre, que des internautes du camp adverse remettent en cause la version officielle pour accréditer l'hypothèse d'une bavure policière. Ces internautes puisent également dans les nombreuses images qui circulent pour appuyer leurs propos. Ainsi, une image partagée par @CiGloFe montre l'incident sous un autre angle (cf. Figure 5). Si la présence de policiers et d'un groupe de personnes aux sols peut laisser penser que les manifestant.es sont tombé.es sous l'effet de la charge, le texte associé dans le tweet assume clairement ce point de vue. L'expression " on voit bien » cherche effectivement à présenter l'interprétation comme une évidence. Dans une seconde publication (cf. Figure 6), @SuperTwitto remet en cause la capacité des images fixes à trancher le différend et partage une vidéo de l'incident en conseillant aux autres internautes d'arrêter de " perdre [leur] temps en publiant une photo sans mouvement pour justifier que Geneviève Legay est tombée toute seule. Y'a une charge, elle est bousculée, elle tombe. Fin du débat ».

En réponse à (i)davduf et iiplace Beauvat

\#legay \#nice \#geneviève on voit bien qu'ils sont trois à tomber sous l'effet direct de la charge. Geneviève. Le mec au sac à dos rouge qui lève sa main. Le photographe juste devant lui.

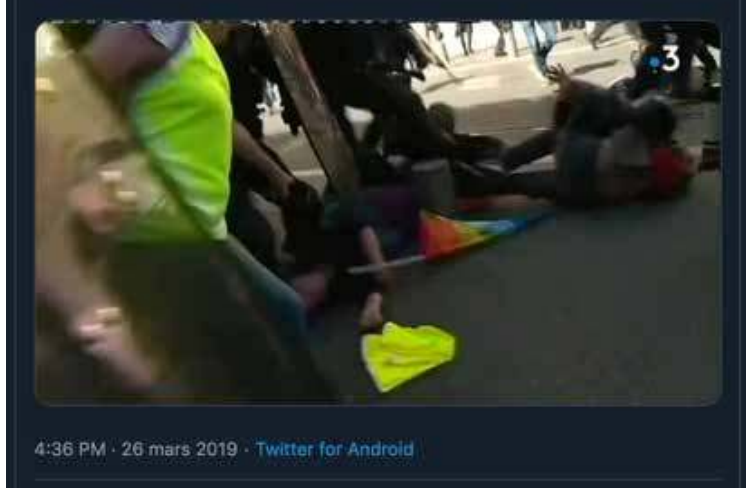

Figure 5 : Capture d'écran d'une vidéo de France 3 pour soutenir une démonstration 


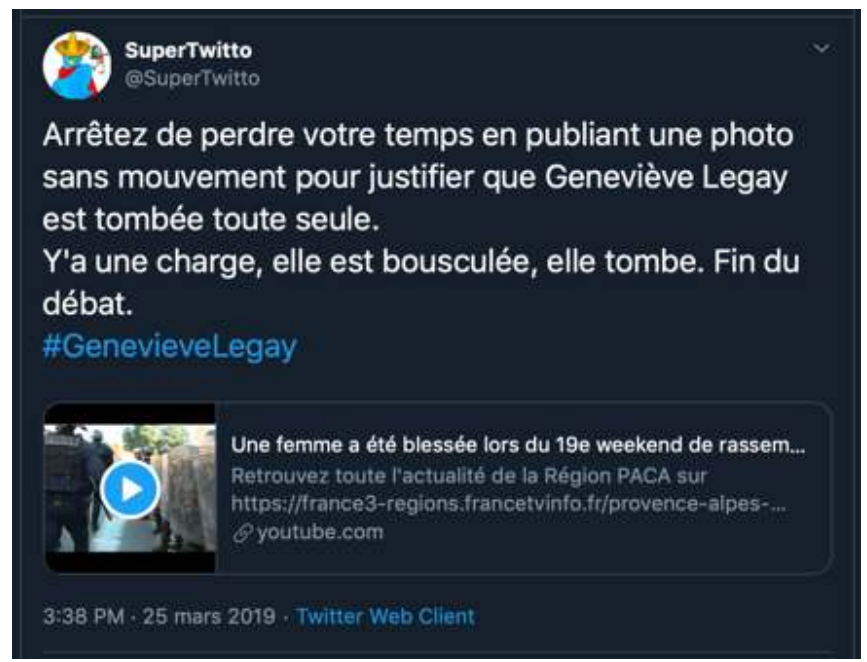

Figure 6 : Publication d'une vidéo hébergée sur YouTube par France 3

Comme en témoignent les propos de @SuperTwitto, des internautes sont confronté·es aux interprétations et aux images mobilisées par le camp opposé. Sur Twitter, les images circulent donc au-delà des homogénéités idéologico-politiques. Ce constat apporte un éclairage nouveau sur les débats concernant les bulles de filtres et les chambres d'écho. L'analyse de notre corpus rend compte de ces nouvelles formes de débat et des affrontements qui se nouent autour des images. Les images ont donc bien une fonction " conversationnelle », pour reprendre l'expression d'André Gunthert (2018) : elles favorisent la discussion entre les deux camps opposés. Mais, le dialogue qui se noue à partir et à propos des images est conflictuel. Les images, en circulant, densifient les échanges et les rendent plus agressifs. Dans l'extrait suivant (cf. Figure 7), deux internautes s'affrontent autour de différentes images de l'incident. D'un côté, @Larrytheblueb dénonce le discours sur la bavure policière quand @KurtetMarvin tente de prouver que Geneviève Legay a bien été agressée par le policier. Chacun des deux camps fait appel à des images pour renvoyer l'adversaire à sa " mauvaise foi » et à la " malhonnêteté intellectuelle » de ses arguments. À partir d'un tweet de @Larrytheblueb dénonçant la manipulation d'extrême-gauche autour de l'affaire, propos appuyé par une photo de la militante au milieu des policiers, un échange de 8 tweets s'enclenche au cours duquel chacun commente les images partagées par l'autre dans le fil de la conversation pour tenter de mettre l'adversaire en défaut. Comme le montre le dernier post de @KurtetMarvin, les images jouent ici directement le rôle d'argument. Il affirme ainsi qu'il a « fait une capture d'écran de cet agent des FDO juste après qu'il ait poussé cette personne. Et elle coïncide parfaitement avec ton image. Il s'agit bien du même agent et la personne qu'il a poussé et qui tenait un GJ dans sa main droite est bien Geneviève Legay ». 


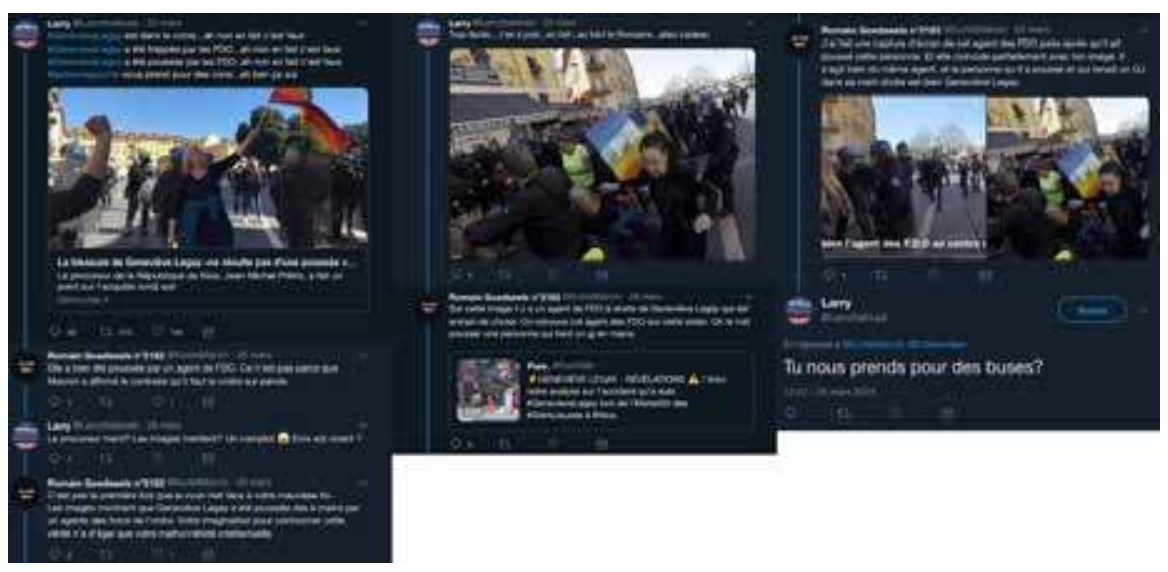

Figure 7 : Confrontation d'images entre deux internautes en désaccord

Aucune image mobilisée sur Twitter ne parvient finalement à véritablement clore le débat en démontrant de manière irréfutable l'absence de bavure policière ou au contraire la responsabilité des forces de l'ordre. Les images sont ainsi moins des preuves en elles-mêmes, comme le revendiquent les internautes, que des supports au débat qui entretiennent la dynamique des échanges. L'impossible consensus au sein de ces échanges conflictuels tient du fait que chaque internaute qui s'exprime publiquement conserve sa position sur l'affaire, malgré les images et les arguments des opposant·es. Ainsi, l'espace public dans lequel les internautes s'expriment peut être compris, moins comme un espace dialogique, où la discussion rationnelle doit aboutir à un commun accord entre les différentes parties, qu'un espace agonistique où l'expression n'a pas vocation à réconcilier des points de vue opposés (Mouffe, 2010).

\section{3. \\ Une montée en généralité axiologique du débat appuyée sur de nouvelles images}

Rapidement cependant, la nature des débats évolue. Face à l'impossibilité de s'accorder sur la responsabilité de la police dans la chute de Mme Legay, les échanges entre les deux camps glissent vers un nouveau débat sur la légitimité des violences policières. Dans son argumentation, chaque camp fait appel à des registres de justifications qui traduisent différentes conceptions du monde (Boltanski et Thévenot, 1991). Dans la confrontation avec d'autres opinions, ces justifications - et les principes et valeurs qui les sous-tendent sont mis à l'épreuve de la discussion. À titre d'exemple, on peut observer que les défenseurs de la légitimité de l'action policière ont tendance à s'inscrire 
dans un registre argumentaire qui fait du respect de l'ordre et de l'État une valeur supérieure. Lors des débats sur Twitter, cette vision du monde se retrouve explicitée et parfois nuancée à travers les débats avec les défenseurs de la thèse de la bavure policière qui analysent l'événement avec leur propre grille où la question de la défense des libertés publiques se trouve en haut de la hiérarchie des valeurs. Ce glissement traduit une montée en généralité des échanges : les internautes réinscrivent l'incident dans leur grille de lecture de l'actualité, ce qui oriente progressivement la discussion vers la controverse plus générale sur la légitimité de la répression policière du mouvement des Gilets Jaunes. Le statut des images mobilisées change également. Celles-ci ont désormais une fonction plus " axiologique » dans la mesure où, au-delà des enjeux de "faire preuve ", elles sont également le support à la confrontation des opinions politiques, des valeurs et des normes mobilisées dans le conflit. Les propriétés techno-sémiotiques de Twitter accentuent ce phénomène.

Ainsi, lorsqu'une image est publiée sur Twitter, elle peut être accompagnée d'un texte, limité à 280 caractères. Cette éditorialisation des contenus permet de créer un nouveau contexte à l'image et d'ouvrir des chemins d'interprétation inédits pour les lecteur-trices du tweet. Nous avons pu observer que ce processus permettait aux internautes de partager leur lecture politique des images. C'est par exemple le cas d'une image qui montre Mme Legay au sol enjambée par un policier (cf. Figure 8). En partageant cette image, @guillau61524 s'inquiète de l'avenir, estimant que I'« on va vers un certain diktat ", et que le droit de manifester est un « droit fondamental ». Pour lui, tenter de justifier «la violence sur des personnes comme Geneviève Legay qui sont loin d'être violentes " par "l'interdiction de manifester » imposée aux Gilets Jaunes est « impressionnant ». Dans un autre tweet, @GaspardAlizan compare l'image de la manifestante blessée à celle du "Manifestant inconnu » qui tenta en 1989 de stopper l'avancée de chars en route pour réprimer les manifestations de la place Tian'anmen à Pékin. La comparaison entre ces deux images fait référence à la démesure de la répression des manifestations, en opposant le pacifisme des manifestant.es à la violence des forces de l'ordre. Le choix de cette comparaison peut également s'expliquer par le fait que la visite du président chinois Xi Jinping à Nice les 24 et 25 mars 2019, a été largement invoquée par les autorités pour justifier de l'interdiction de manifester dans certains lieux à $\mathrm{Nice}^{12}$. 

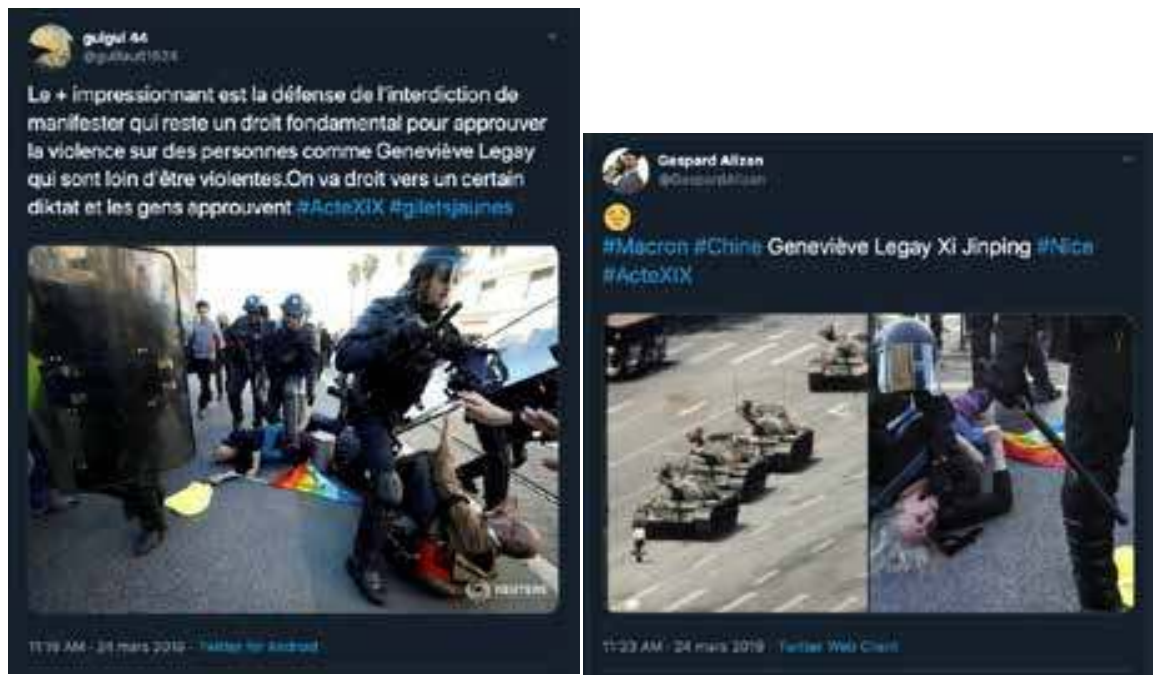

Figure 8 : Images mobilisées pour dénoncer la dérive autoritaire de l'État

La montée en généralité est également exprimée par le biais de montages photos réalisés par les internautes (cf. Figure 9). Ces images font référence aux blessures provoquées par les forces de l'ordre depuis le début du mouvement des Gilets Jaunes et cadrent les échanges au-delà de la seule blessure de la militante, qui est absente des images, mais dont le nom apparaît dans les textes d'accompagnement. Ainsi, @3Solidaires publie une série de photos de Gilets Jaunes blessés qu'il associe directement à l'incident. Dans la même logique, @tropical_boy, lui, rebondit sur l'expression des « regrets » du policier ayant poussé Mme Legay, afin de revendiquer l'attente d'une telle compassion de la part de tou-tes les autres policier-ères qui ont blessé des Gilets Jaunes. II appuie son propos en publiant un montage présentant de nombreux Gilets Jaunes blessés. 


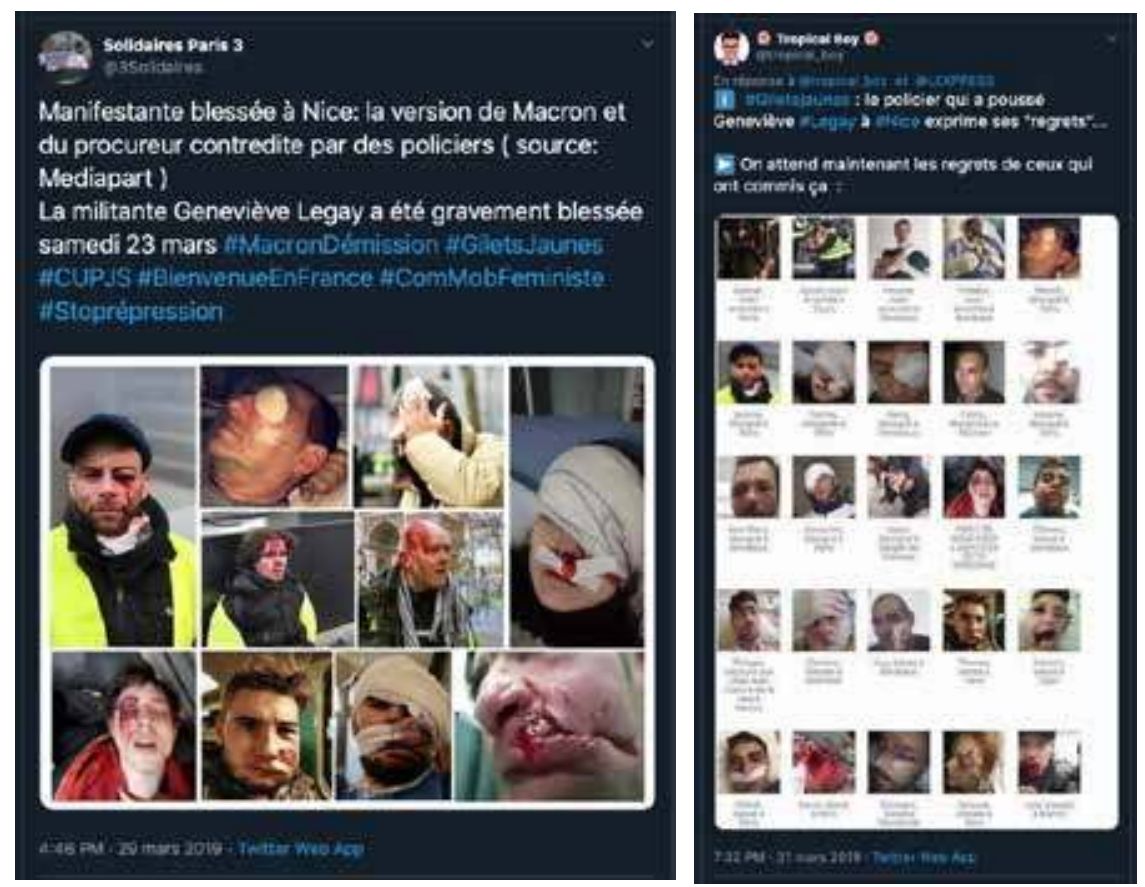

Figure 9 : Plusieurs blessé·es Gilets Jaunes associé·es à l'affaire Legay

À l'inverse, les soutiens aux forces de l'ordre ont progressivement cherché à mettre en avant la violence des Gilets Jaunes pour légitimer le niveau de répression du mouvement (cf. Figure 10). @MinipouceLyon publie ainsi une photo barrée d'un « \#StopGiletsJaunes » représentant des véhicules blindés de la gendarmerie faisant face à des objets enflammés. Dans son tweet, l'internaute s'indigne d'être " pris en otages par des émeutes » chaque weekend et du non « respect des lois républicaines » par les Gilets Jaunes. @Faure05Faure mobilise l'image d'un scooter enflammé à proximité de manifestant·es, certain·es portant un gilet jaune. L'internaute critique la plainte déposée par celle qui n'a pas respecté la loi en manifestant dans un lieu interdit, et compare les Gilets Jaunes dans leur ensemble à des " casseurs », des « voyous " et des " délinquants ». 


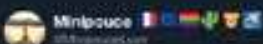

couttale ir lo droit do circuler, commercer, to respect des lois républicaines, ete..

Si des limites ont été mises sur certains lieux. c'est aussi pour que les autres pulssent vivie sans atre ts las wo pris en otages par des ermeutes (P)

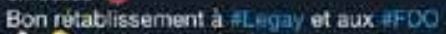

A

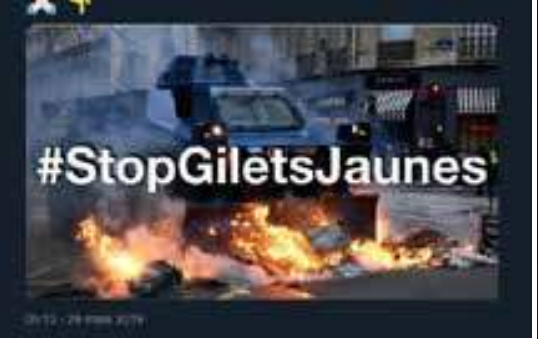

2. pean fauer

Qy

VIVA LA REVOUUTION, "c'est un digat collatéral de la revolution" les man'is NON déclarés cest normal, is respect de la loi c'est pour les autres, wha les voyous, les delinquants, les casseurs en $6 J$, ON NE RESPECTE PAS LA LOI MAIS ON PORTE PLANTE "Genevieve

Legay"

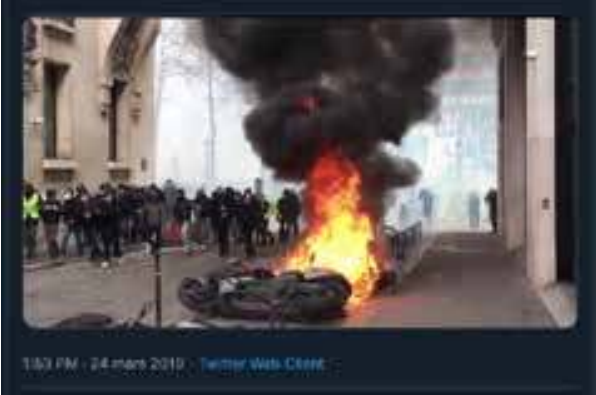

Figure 10 : Deux images-axiologiques mobilisées par des « anti-Gilets Jaunes »

La fonctionnalité de Twitter qui encourage les interactions entre internautes et l'interpellation d'autres comptes a également contribué à accorder aux images un rôle pivot dans le basculement du débat. Elles ouvrent la possibilité de communiquer avec autrui au-delà des bulles de filtre, dans des espaces agonistiques où la blessure de Geneviève Legay est en réalité l'occasion d'un affrontement entre « contre-mondes » (Carbou, 2015a).

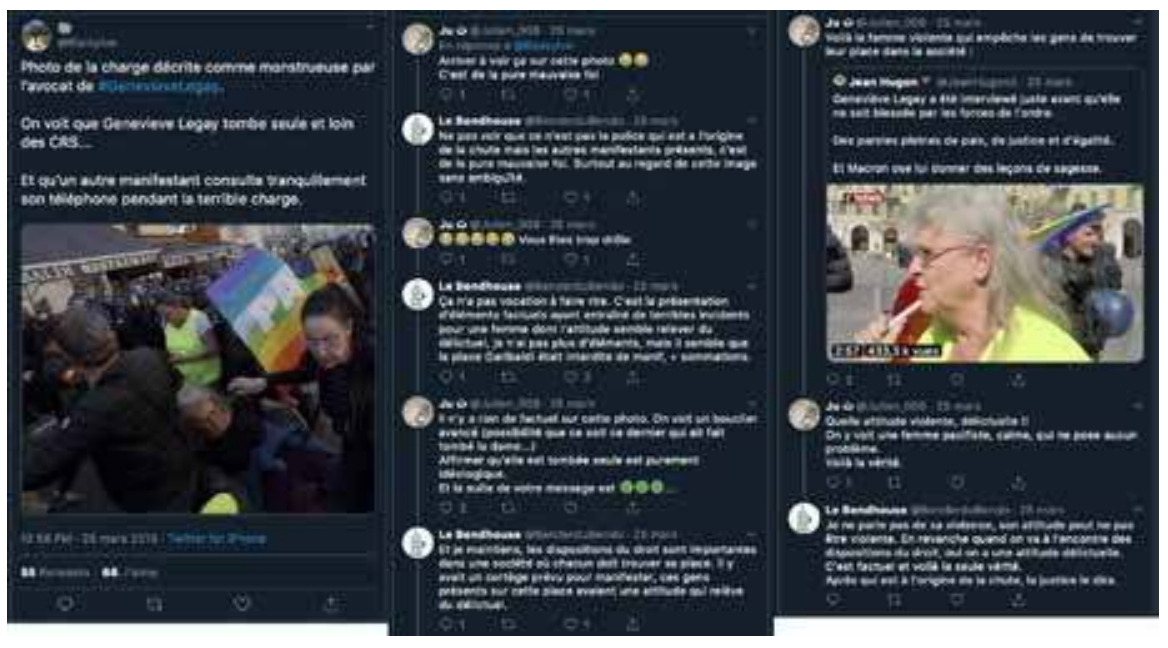

Figure 11 : Une vidéo " pivot » qui installe le débat dans une logique axiologique 
Un échange du 25 mars 2019 rend compte de cette dynamique d'échange qui contribue à la réorientation du débat (cf. Figure 11). Sur Twitter, l'interaction se matérialise principalement par un ensemble de réponses à un tweet. Pour @Ripisylve qui publie l'image classiquement mobilisée par les défenseurs des forces de l'ordre, "On voit que Geneviève Legay tombe seule et loin des CRS ". Un échange fourni s'engage. 39 réponses directes auxquelles il faut ajouter les nombreuses réponses à ces réponses. Suite à ce premier post, @Julien_008 met en avant la mauvaise foi de la publication de @Ripisylve, ce à quoi un troisième internaute, @BenderduBendo, en défense des forces de l'ordre, rétorque que l'image est pourtant "sans ambiguïté », qu'elle présente des "éléments factuels ayant entraîné de terribles incidents pour une femme dont l'attitude semble relever du délictuel ». Prolongeant l'échange, @Julien_008 estime dans un nouveau tweet qu'il n'y a pourtant « rien de factuel sur cette photo ». Pour lui, " affirmer qu'elle est tombée seule est purement idéologique ». Face au maintien du désaccord, le débat se déplace et monte en généralité : @BenderduBendo fait alors appel aux « dispositions du droit » et revient à la charge sur l'attitude des manifestant·es "qui relève du délictuel ». @Julien_008 relaye une vidéo de l'interview de Geneviève Legay par CNews pour insister sur ses " paroles pleines de paix, de justice et d'égalité ». Il met alors en avant le " pacifisme » et le « calme » de la manifestante, pour mieux se moquer de son adversaire dans un second tweet : "Quelle attitude violente, délictuelle !! ». L'échange prend fin avec un message de @BenderduBendo qui clôt la discussion en nuançant son propos : il insiste désormais sur le fait que, dans le respect du droit, c'est la justice qui statuera de « qui est à l'origine de la chute».

\section{Conclusion}

Évidemment, les échanges sur Twitter n'ont pas permis de déterminer à coup sûr les responsabilités dans l'incident qui a conduit à la blessure de Geneviève Legay. Cependant, nous avons pu constater que la dynamique dialogique s'est progressivement transformée en "épreuve démocratique » où la légitimité de l'action des forces de l'ordre dans le mouvement des Gilets Jaunes a été débattue. L'enquête que nous avons proposée a permis d'identifier les ressorts de cette montée en généralité des échanges. Nous avons ainsi pu montrer que les propriétés communicationnelles des RSN (capacité à susciter de l'interaction, conflictualité forte...) contribuent à transformer la manière dont les citoyens se saisissent d'un sujet de société et le problématisent.

Dans cette nouvelle culture du débat public, les manières d'argumenter évoluent également. Les ressources médiatiques, à l'instar des images, jouent désormais un rôle clé. Si elles constituent un appui à l'argumentation pour «faire preuve ", elles jouent également un rôle plus direct de point de rencontre entre les différentes communautés qui vont réagir - pour soutenir ou critiquer - le discours que l'image permet de construire. Nous avons également observé que la circulation des images contribuait à emmener la controverse sur le ter- 
rain des valeurs, dans la mesure où elles sont mobilisées par les participant.es pour justifier leur positionnement. En ce sens, elles peuvent être considérées comme des " objets-frontières » (Stars et Griesemer, 1989) à l'interface entre des mondes sociaux et des acteurs ayant des perspectives différentes.

Comprendre ces mécanismes nous a permis d'interroger avec plus de précision le statut argumentatif des images dans les controverses en ligne. Nous avons ainsi constaté une transformation des registres rhétoriques mobilisés avec un glissement du logos (l'appel à la raison) vers le pathos (l'appel aux émotions). Dans un premier temps, les « images-preuves " semblent pouvoir faire tenir à elles seules la démonstration dans une logique présentée comme objective. Dans ce cadre, l'argumentation textuelle est assez pauvre. Les twittos font seulement référence aux images (avec des expressions du type " on voit bien que... »), en considérant celles-ci comme étant autosuffisantes pour exprimer une vérité indubitable. On ne cherche pas tant à convaincre qu'à démontrer grâce aux images. Le texte qui leur est associé dans les tweets sert alors essentiellement à " fixer la chaîne flottante des signifiés » afin de rompre avec le caractère polysémique des images et limiter les interprétations possibles en ancrant photos et vidéos dans une démonstration donnée (Barthes, 1964).

Progressivement, cette logique d'enquête, où l'on cherche à démêler le vrai du faux, laisse sa place à une seconde phase de la controverse. Faute de pouvoir trancher et imposer leur point de vue, les participant-es aux échanges vont avoir tendance à mobiliser les images pour soutenir une argumentation plus générale exprimant un point de vue ou un ensemble de valeurs. Ces images sont plus connotées et suggestives, dans la mesure où elles viennent soutenir un propos à vocation plus universelle, délivré par des énonciateurs qui assument davantage une posture idéologiquement marquée. On cherche alors moins à exposer la vérité qu'à susciter l'émoi, parfois de façon ostentatoire, de manière à mettre en avant des analogies et des similitudes avec d'autres événements (à l'instar de la fameuse image du manifestant de la place Tian'anmen face aux chars) afin de créer des symétries émotionnelles. Les textes associés invitent alors au relai d'opinions et à la mise en circulation d'affects.

D'une séquence à l'autre, c'est progressivement une discussion contradictoire sur le thème des violences policières et de la légitimité de l'intervention des forces de l'ordre qui se met en place. Cette dynamique ouvre des espaces inédits de confrontation de visions du monde - les espaces de commentaires au sein des RSN - qui nous permettent d'interroger la manière dont se construit en ligne la critique citoyenne des institutions et des médias dominants. Ces espaces de commentaires constituent alors des espaces de réflexivité démocratique qui accueillent une réflexion citoyenne profonde et axiologique sur l'état actuel de la démocratie, marquée par un contexte où la répression des mobilisations sociales en France est toujours plus préoccupante. 
Bibliographie

Angenot M. (2008). Dialogues de sourds : traité de rhétorique antilogique, Paris, Mille et une nuits.

Badouard R. (2017). Le désenchantement de l'Internet : désinformation, rumeur et propagande, Limoges, Fyp éditions.

Barthes R. (1964). « Rhétorique de l'image ». In Communications, vol. 4, p. 40-51.

Boëx C. (2012). « Montrer, dire et lutter par l'image. Les usages de la vidéo dans la révolution en Syrie ". In Vacarme, vol. 61, p. 118-131.

Boltanski L., Thévenot L. (1991). De la justification. Les économies de la grandeur, Paris, Gallimard.

Campion B. (2016). " Dynamiques de construction et instrumentalisation de la légitimité dans les débats en ligne relatifs au réchauffement climatique ». In Questions de communication, vol. 30, $n^{\circ} 2$, p. 159-179.

Carbou G. (2015a). « Des contre-discours aux contre-mondes : l'exemple des commentaires d'internautes autour de l'accident de Fukushima ». In Semen, vol. 39, p. 81-98.

Carbou G. (2015b). «Presse en ligne et communautés cognitives.

Les sections commentaires d'Atlantico.fr et de l'Humanité.fr durant I' 'affaire DSK' ». In Réseaux, vol. 193, n 5, p. 193-223.

Cardon D. (2010). La démocratie Internet : promesses et limites, Paris, Seuil.

Cardon D. (2019). Culture numérique, Paris, Les Presses de Sciences Po.

Cardon D., Granjon F. (2010). Médiactivistes, Paris, Les Presses de Sciences Po.

Dalibert M., Lamy A., Quemener N. (2016). « Introduction : Circulation et qualification des discours. Conflictualités dans les espaces publics (1) ». In Études de communication, vol. 47, p. 7-20.

Dodier N. (1999). « L'espace public de la recherche médicale. Autour de l'affaire de la ciclosporine ". In Réseaux, vol. 95, n 4, p. 107-154.

Douyère D. (2019). « L'image dont on parle, l'image avec laquelle on parle. De la colonisation iconique de la conversation ". In Questions de communication, vol. 35, $n^{\circ} 1$, p. 137-151.

Entman R. M. (1993). “Framing: Toward Clarification of a Fractured Paradigm". In Journal of Communication, vol. $43, n^{\circ} 4$, p. 51-58.

Fraser N. (2001 [1992]). « Repenser la sphère publique : une contribution à la critique de la démocratie telle qu'elle existe réellement ". In Hermès, vol. 31, n 3, p. 125-156. 
Gillespie T. (2014). "The Relevance of Algorithms". In Gillespie T., Boczkowski P. J., Foot K. A. (dirs.), Media Technologies: Essays on Communication, Materiality, and Society, Cambridge, The MIT Press, p. 167-93.

Gamson W. A., Modigliani A. (1989). "Media Discourse and Public Opinion on Nuclear Power: A Constructionist Approach". In American Journal of Sociology, vol. 95, p. 1-37.

Granjon F. (2018). « Mouvements sociaux, espaces publics et usages d'Internet ». In Pouvoirs, vol. 164, $n^{\circ} 1$, p. 31-47.

Gunthert A. (2018). «La visibilité des anonymes. Les images conversationnelles colonisent l'espace public ». In Questions de communication, vol. $34, \mathrm{n}^{\circ} 2$, p. 133-154.

Habermas J. (1962). L'espace public, Paris, Éditions Payot.

Jenkins H. (2006). Convergence Culture: Where Old and New Media Collide, New York, New York University Press.

Jammet T. (2018). « L'activité de community management à l'épreuve de l'architecture algorithmique du web social ». In Réseaux, vol. 212, n 6, p. 149-178.

Julliard V. (2015). « Les apports de la techno-sémiotique à l'analyse des controverses sur Twitter ». In Hermès, vol. 73, n³, p. 191-200.

Julliard V. (2016). «\#Theoriedugenre : comment débat-on du genre sur Twitter? ». In Questions de communication, vol. 30, p. 135-157.

Julliard V. (2017). «'Théorie du genre', \#theoriedugenre : stratégies discursives pour soustraire la 'différence des sexes' des objets de débat ». In Études de communication, vol. 48, p. 111-136.

Lemieux C. (2007). «À quoi sert l'analyse des controverses?". In Mil neuf cent. Revue d'histoire intellectuelle, vol. 25, n 1, p. 191-212.

Mabi C. (2016). « Luttes sociales et environnementales à l'épreuve du numérique : radicalité politique et circulation des discours ". In Études de communication, vol. 47, p. 111-130.

Milan S. (2013). Social Movements and Their Technologies: Wiring Social Change, Basingstoke (RoyaumeUni), Palgrave Macmillan.

Mouffe C. (2010). "Politique et agonisme ". In Rue Descartes, vol. $67, n^{\circ} 1$, p. 18-24.

Pariser E. (2011). The filter bubble: what the Internet is hiding from you, New York, Penguin Press.

Rebillard F. (2017). « La rumeur du PizzaGate durant la présidentielle de 2016 aux États-Unis : Les appuis documentaires du numérique et de I'Internet à l'agitation politique ". In Réseaux, vol. 202-203, n 2, p. 273-310.

Rennes J. (2007). « Analyser une controverse. Les apports de l'étude argumentative à la science politique ». In Bonnafous S., 
Temmar M. (dirs.), Analyse du discours et sciences humaines et sociales, Paris, Ophrys, p. 91-107.

Riboni U. L. (2015). « Filmer et rendre visible les quartiers populaires dans la Tunisie en révolution ". In Sciences de la société, vol. 94, p. 121-136.

Riboni U. L. (2019). « Images anonymes, registres de visibilité et espace(s) public(s) ». In Questions de communication, vol. 35, n¹, p. 153-169.

Rieder B., Smyrnaios N. (2012). «Pluralisme et infomédiation sociale de l'actualité : le cas de Twitter $»$. In Réseaux, vol. 176, n 6, p. 105-139.

Schradie J. (2019). The Revolution That Wasn't: How Digital Activism Favors Conservatives, Cambridge, Harvard University Press.

Snow D. A., Rochford E. B., Worden S. K., Benford R. D. (1986). "Frame Alignment Processes, Micromobilization, and Movement Participation". In American Sociological Review, vol. 51, n 4, p. 464-481.

Stars S. L., Griesemer J. R. (1989). "Institutional Ecology, 'Translation' and Boundary Objects: Amateurs and Professionals in Berkeley's Museum of Vertebrate Zoology, 1907-39", In Social Studies of Science, vol. 19, n³, p. 387-420.

Sustein C. R. (2009). Republic. com, Princeton and Oxford, Princeton University Press.
Voirol O. (2005). « Les luttes pour la visibilité. Esquisse d'une problématique ». In Réseaux, vol. 129-130, n 1-2, p. 89-121. 
